\title{
Infrared and visible image fusion based on shiftable complex directional pyramid transform and SUSAN edge detector
}

\author{
${ }^{1}$ Defa Hu, ${ }^{2}$ Hailiang Shi and ${ }^{1}$ Weijin Jiang \\ ${ }^{1}$ Key Laboratory of Hunan Province for Mobile Business Intelligence, Hunan \\ University of Commerce, Changsha 410205, Hunan, China, hdf666@163.com \\ ${ }^{2}$ College of Mathematics and Information Science, Zhengzhou University of Light \\ Industry, Zhengzhou 450002, Henan, China, 3812603@qq.com
}

Received: 08.07 .2018

\begin{abstract}
We propose an algorithm for fusing infrared and visible images, which is based on a shiftable complex directional pyramid transform and a SUSAN edge detector. When fusing the low-pass sub-band coefficients, we employ a weightedaveraging rule based on local normalized energy. When fusing the directional highpass sub-bands coefficients, the SUSAN edge detector is utilized to generate a decision map and guide the fusion process. In order to reduce the computational complexity, we calculate the SUSAN edge response only once directly for each source image. We evaluate our fusion algorithm on a standard TNO Image Fusion Dataset, using comparisons with a number of traditional fusion algorithms. The experimental results testify that our algorithm is efficient and feasible. Moreover, it is superior to the traditional algorithms from the viewpoints of subjective evaluation and objective fusion metrics.
\end{abstract}

Keywords: image fusion, infrared and visible images, shiftable complex directional pyramid transform, SUSAN edge detector

PACS: $42.30 . \mathrm{Va}$

UDC: 535.8

\section{Introduction}

Multi-sensor images usually provide complementary and redundant information about the same scene, and image fusion aims to merge these information peaces into a single image to offer a more complex and detailed representation. In surveillance applications, visible sensors mainly reflect only the rich appearance information. On the contrary, infrared sensors reflect basically the profile information on objects by capturing their thermal radiation. They are not affected by any changes in light or disguise, thus enabling to discover the targets and work day and night [1]. Therefore, fusion of those different information components is needed for automatic detection and localization of the targets [1].

Up to now, many fusion algorithms have been proposed for infrared and visible images. Generally they can be categorized into three groups based on different fusion mechanisms: pixellevel, feature-level and decision-level [2]. Among these algorithms, a pixel-level fusion is the most commonly used. This is due to such its advantages as easy implementation and high computational efficiency [2]. The pixel-level algorithms are based on a multi-scale transform (MST). The wellknown MST tools include a Laplacian pyramid transform (LPT) and a discrete wavelet transform (DWT). Because the DWT is superior to the LPT, in particular with respect to localization and orientation, the appropriate fusion algorithm is generally superior to that associated with the LPT. However, the DWT for 2D signals cannot represent discontinuity of a 'line' or a 'curve' efficiently 
and, moreover, it can only capture a limited directional information [3]. Contourlet transform (CT) [2] is a 'true' sparse 2D representation which is implemented by coupling the LPT with a directional filter bank (DFB). If compared to the DWT, the CT is characterized by multi-scaliness and localization and has the characteristics of multi-directing and anisotropy. However, like the DWT, the CT lacks a shift-invariance [3].

Somewhat later, a nonsubsampled contourlet transform (NSCT) has become widely used as a tool for multi-scale analysis. Nonetheless, it cannot reflect well complex spatial structures along different directions, due to a fixed DFB in directional decomposition [4]. Moreover, this technique is time-consuming. To address this problem, Nguyen et al. have proposed a shiftable complex directional pyramid transform (SCDPT) [5], which is considered as a complex shiftable version of the CT aimed at image fusion [3]. The SCDPT has many ideal characteristics, such as a shiftable sub-band, arbitrary high directionality and availability of information concerned with the phase $[5$, 6]. In image fusion, the SCDPT can provide more structural information and reduce the impacts of mis-registration because of its shift-invariance property [3].

In the present work we propose an algorithm for fusing infrared and visible images, which is based on the SCDPT and a SUSAN edge detector. In the light of characteristics of human vision system and the SCDPT, we discuss in detail a weighted-averaging fusion rule based on the local normalized energy for low-pass sub-bands and a maximum-choosing fusion rule for high-pass subbands based on the SUSAN edge detector. Several experiments have been performed to show validity and efficiency of the fusion algorithm suggested by us.

The rest of the article is organized as follows. Section 2 briefly reviews a theory of SCDPT. Section 3 describes in detail the proposed algorithm for fusing infrared and visible images. The experimental results and discussion are presented in Section 4, and the conclusion is drawn in Section 5 .

\section{Shiftable complex directional pyramid transform}

Below we review in brief a theory of SCDPT used in the subsequent sections. The SCDPT uses the multi-scale filter bank (FB) and the DFB in its two decomposition phases. The multi-scale FB is made of undecimated two-channel FB and an iterated 2D multi-resolution FB in the low-pass phase. The structure of the SCDPT is shown in Fig. 1. A signal $x(n)$ is decomposed by the undecimated two-channel FB into $L_{0}(n)$ and $R_{0}(n)$ signals. $L_{0}(n)$ can cause aliasing in the next stage,

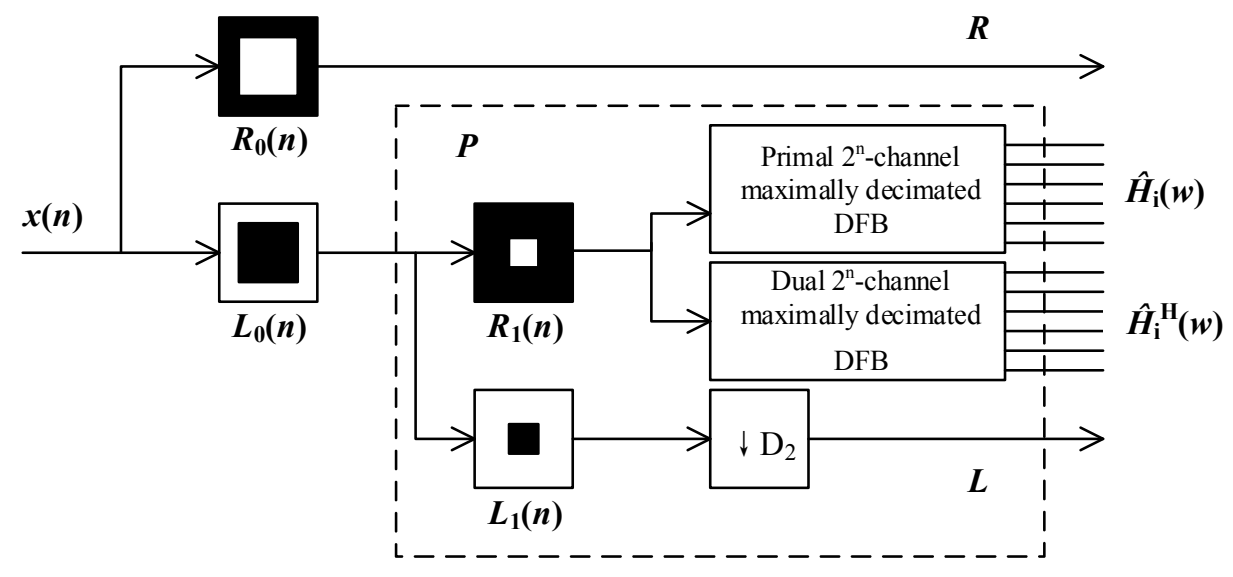

Fig. 1. Structure of SCDPT. 
and $R_{0}(n)$ generates a residual image. The $L_{0}(n)$ signal is brought into a new round of multiresolution decomposition, resulting in $L_{1}(n)$ and $R_{1}(n)$. This generates low-pass and high-pass signal components. Then the high-pass component is further brought into a dual-tree directional FB to generate real and imaginary parts of complex directional sub-bands $\hat{H}_{i}(w)$ and $\hat{H}_{i}^{H}(w)$, whereas the corresponding low-frequency component is decimated by $D_{2}=\left[\begin{array}{ll}2 & 0 \\ 0 & 2\end{array}\right]$, thus generating a low-pass sub-band. This decomposition performed in a block $\mathrm{P}$ is iterated to form a pyramid. The pyramidal dual-tree DFB consists of a shiftable pyramidal FB and a dual-tree $2^{n}$ channel DFB to provide multi-scale decomposition. The block $P$ in Fig. 1 illustrates the first level of the pyramidal dual-tree DFB [5].

\section{Image fusion algorithm}

\subsection{Fusion framework}

Now we present the framework of our fusion algorithm. Consider two perfectly registered infrared and visible images $A$ and $B$, and a fused image $F$. The fusion algorithm consists of the following steps.

(1) Perform $J$-level SCDPT on A and B to obtain a single low-pass sub-band and a series of high-pass sub-bands for each decomposition level and each direction. The corresponding coefficients can be represented as

$$
\left\{C_{J}^{A}(x, y), C_{j, \theta}^{A}(x, y)\right\},\left\{C_{J}^{B}(x, y), C_{j, \theta}^{B}(x, y)\right\},
$$

where $1 \leq j \leq J$ denotes the $j$ th scale and $\theta$ the direction. Note that $C_{j, \theta}^{I}(x, y)(I=A, B)$ are complex directional sub-band coefficients, whose real parts come from the primal branch and imaginary ones from the dual branch of the complex DFB [6].

(2) Perform a weighted-averaging fusion of the low-pass sub-bands, basing on the local normalized energy to obtain $C_{J}^{F}(x, y)$.

(3) Choose a maximum for the fusion of the high-pass sub-bands, basing on the SUSAN edge detector to obtain $C_{j, \theta}^{F}(x, y)$.

(4) Use the inverse SCDPT and the fusion coefficients $\left\{C_{J}^{F}(x, y)\right.$ and $\left.C_{j, \theta}^{F}(x, y)\right\}$ to obtain $F$.

\subsection{Energy-based fusion of local normalized low-pass sub-bands}

The low-pass sub-bands contain the most of the energy of the source image. It is especially typical for the infrared and night-time visible images, of which sensors are sensitive to temperature and illumination, respectively. Then the image features and the structural information are usually complementary, being reflected in pixel luminance. Therefore we suggest using a local normalized energy as an activity measure in order to capture that information. The measure is defined as

$$
\operatorname{LNE}(x, y)=\frac{1}{|R|} \sum_{i, j \in R}\left(C_{J}^{I}(i, j)\right)^{2},
$$

where $|R|$ implies the size of the local region $R$ of image $I$, which is centred at $(x, y)$, and $(i, j)$ is the pixel position in $R$.

Notice that the features and the structural information of the same object, which are provided by the infrared and visible images, are not always complementary. For example, an electric lamp 
has both high temperature and high illumination, hence the corresponding features in the two type images are both prominent and redundant, and they can have different structural information. To distinguish complementary and redundant information and measure the corresponding proportions, here we adopt the weight-averaging fusion rule. The weights are calculated as

$$
w_{A}(x, y)=\frac{L N E_{A}(x, y)}{L N E_{A}(x, y)+L N E_{B}(x, y)}, w_{B}(x, y)=1-w_{A}(x, y) .
$$

With the above local weights, the fused low-pass coefficients can be obtained as [6]

$$
C_{J}^{F}(x, y)=w_{A}(x, y) \cdot C_{J}^{A}(x, y)+w_{B}(x, y) \cdot C_{J}^{B}(x, y) .
$$

\subsection{Fusion of high-pass sub-bands based on the SUSAN operator}

SUSAN is an approach worked out for the low-level image processing. The basis of the SUSAN is associating of each image pixel with the brightness of its local area. Here the local area called as a 'Univalue Segment Assimilating Nucleus' (or USAN) contains much structural information. Then the image features can be measured by the size, centroid and the second moments of this nucleus [7].

The SUSAN edge detector described in this section follows a traditional SUSAN algorithm. It is implemented using a circular mask, whose usual radius is 3.4 pixels. The brightness comparison takes place among each pixel within the mask and a central pixel, which can be expressed as follows:

$$
c\left(\vec{r}, \vec{r}_{0}\right)=\exp \left(-\left(\frac{I(\vec{r})-I\left(\vec{r}_{0}\right)}{t}\right)^{6}\right),
$$

where $\vec{r}_{0}$ and $\vec{r}$ are the positions of respectively the central pixel and each pixel within the mask, $I(\cdot)$ means the brightness, $t$ the threshold and $c$ the result of comparison.

The summation of $n$ comparison results (with $n$ being the number of pixels within the mask) is given by

$$
n\left(\vec{r}_{0}\right)=\sum_{\vec{r}} c\left(\vec{r}, \vec{r}_{0}\right) .
$$

Then a fixed geometric threshold $g$ is set as

$$
g=3 n_{\max } / 4
$$

where $n_{\max }$ is the maximum $n$ value. After that, the initial edge response $R\left(\vec{r}_{0}\right)$ can be calculated as

$$
R\left(\vec{r}_{0}\right)=\left\{\begin{array}{l}
g-n\left(\vec{r}_{0}\right), \text { if } n\left(\vec{r}_{0}\right)<g \\
0, \text { otherwise }
\end{array} .\right.
$$

Generally speaking, the smaller the area of the univalue segment assimilating nucleus, the larger the edge response is [7].

High-pass sub-bands obtained using the SCDPT include usually the edges along various directions, which is in accord with the ability of SUSAN edge detectors. For preserving more edge information, the maximum-choosing fusion rule based on the SUSAN edge detector is adopted for the high-pass sub-band fusion. In the meantime, to reduce drastically the computational complexity, the SUSAN edge response is calculated only once directly on each source image. In the high-pass sub-bands fusion process, the response can be appropriately re-sampled as a decision map according to the size of the high-pass sub-band: 


$$
C_{j, \theta}^{F}(x, y)=\left\{\begin{array}{l}
C_{j, \theta}^{A}(x, y), \text { if } r s\left(R^{A}(x, y)\right) \geq r s\left(R^{B}(x, y)\right) \\
C_{j, \theta}^{B}(x, y), \text { otherwise }
\end{array},\right.
$$

where $r s(\cdot)$ denotes the resample operator.

\section{Experimental results and discussion}

In this section, we test the performance of our fusion algorithm on a publicly available dataset and compare it with several traditional fusion algorithms. The test images are referred to as 'two men', 'tank', 'bunker', 'lake' and 'soldier'. All of them come from a TNO Image Fusion Dataset [8]. The sizes of the images are $576 \times 768$, except for the 'tank' image for which we have the size $352 \times 472$.

We have used for a comparison LPT-based, DWT-based, CT-based and NSCT-based fusion algorithms, as well as the algorithm based upon a nonsubsampled shearlet transform (NSST). In case of all of these algorithms, the classical averaging and maximum-choosing rules have been used for the fusion of the low-pass and high-pass sub-bands with three decomposition levels. Standard default parameters have been used for the LPT- and DWT-based algorithms. The bases of the CT and the NSCT are '9-7' for the pyramid filter and 'pkva' for the directional filter. Finally, the basis used in the NSST is 'maxflat'.

The performance of the fusion algorithms can be evaluated either subjectively or objectively. The mathematical definitions of a number of objective fusion metrics adopted in the field are as follows.

(1) Average gradient measures clarity of the image, being defined as

$$
A G(F)=\frac{1}{(m-1)(n-1)} \cdot \sum_{i=1}^{m-1} \sum_{j=1}^{n-1}\left((F(i, j)-F(i+1, j))^{2}+(F(i, j)-F(i, j+1))^{2}\right)^{\frac{1}{2}},(10
$$

where $F(i, j)$ corresponds to the fused image.

(2) Information entropy [9] is given by

$$
I E(F)=-\sum_{i=0}^{L-1} p(i) \log _{2} p(i),
$$

where $L$ is the gray-scale level and $p(i)$ the marginal probability distribution function for $F$.

(3) Universal image quality index $Q_{0}$ is calculated between the infrared image $A$ and the fused image $F[10]$ :

$$
Q_{0}(A, F)=\frac{\sigma_{A F}}{\sigma_{A} \sigma_{F}} \cdot \frac{2 \mu_{A} \mu_{F}}{\mu_{A}^{2}+\mu_{F}^{2}} \cdot \frac{2 \sigma_{A} \sigma_{F}}{\sigma_{A}^{2}+\sigma_{F}^{2}},
$$

where $\mu_{A}$ and $\mu_{F}$ are respectively the averages of $A$ and $F, \sigma_{A}$ and $\sigma_{F}$ are respectively the variances of $A$ and $F$, and $\sigma_{A F}$ the appropriate covariance.

(4) Structural similarity index measure [11] for the infrared image $A$ and the fused image $F$ is given by

$$
\operatorname{SSIM}(A, F)=\frac{\left(2 \mu_{A} \mu_{F}+C_{1}\right)\left(2 \sigma_{A F}+C_{2}\right)}{\left(\mu_{A}^{2}+\mu_{F}^{2}+C_{1}\right)\left(\sigma_{A}^{2}+\sigma_{F}^{2}+C_{2}\right)},
$$

where $C_{1}$ and $C_{2}$ are constants introduced to avoid instability. Actually, the structural similarity index is a modified version of $Q_{0}$.

(5) Normalized mutual information $Q_{M I}[12]$ is defined as

$$
Q_{M I}=2\left(\frac{M I(A, F)}{H(A)+H(F)}+\frac{M I(B, F)}{H(B)+H(F)}\right),
$$

where 


$$
\begin{aligned}
& M I(I, F)=H(I)+H(F)-H(I, F), \quad H(I)=-\sum_{i} p(i) \log _{2} p(i), \\
& H(F)=-\sum_{i} p(f) \log _{2} p(f), \quad H(I, F)=-\sum_{i, f} p(i, f) \log _{2} p(i, f),
\end{aligned}
$$

and $I=A, B$. Here $p(i, f)$ is the joint probability distribution function for $I$ and $F$, whereas $p(i)$ and $p(f)$ are the marginal probability distribution functions for $I$ and $F$, respectively. Correspondingly, $H(I, F)$ is the joint entropy, and $H(I)$ and $H(F)$ are the marginal entropies.

(6) Tsallis entropy $Q_{T E}$ is represented as [12]

$$
Q_{T E}=\frac{I^{q}(A, F)+I^{q}(B, F)}{H^{q}(A)+H^{q}(B)-I^{q}(A, B)},
$$

where

$$
I^{q}(I, F)=\frac{1}{1-q}\left(1-\sum_{i, j} \frac{h_{I F}(i, j)^{q}}{h_{F}(j)^{q-1} h_{I}(i)^{q-1}}\right),
$$

$q$ is a real value $(q \neq 1), h_{A F}(i, j)$ the normalized joint gray-scale histogram of the images $I(I=A, B)$ and $F$, and $h_{I}(i)$ the normalized marginal histogram of the image $I(I=A, B, F)$.

We first give a subjective analysis of the performance of different image-fusion algorithms. Among the five image sets mentioned above, we have chosen the 'two men' to conduct the experiment (see Fig. 2a, b). Here panels (a) and (b) correspond respectively to the visible image (describing mainly the background) and the infrared image (highlighting the two persons as the targets). The fusion results displayed in Fig. 2c-h refer sequentially to the LPT-based, DWT-based, CT-based, NSCT-based, NSST-based and our fusion algorithms.

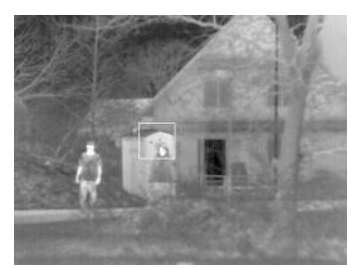

(a)

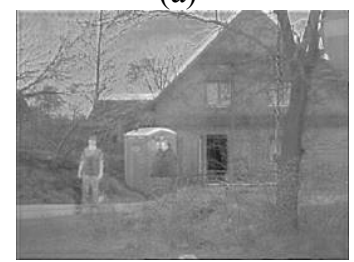

(e)

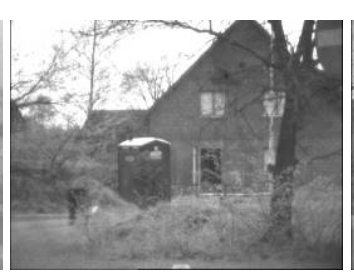

(b)

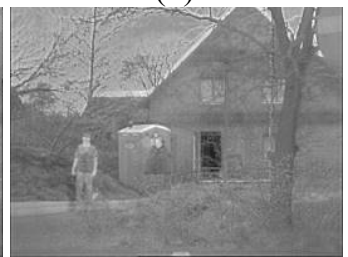

(f)

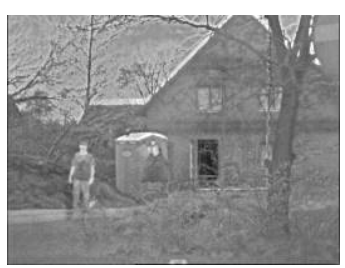

(c)

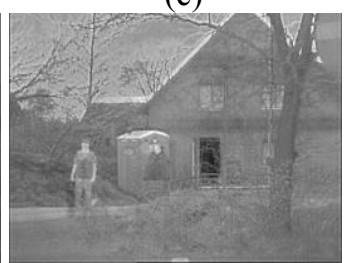

(g)

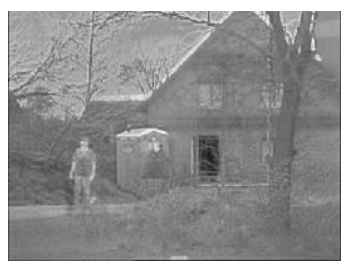

(d)

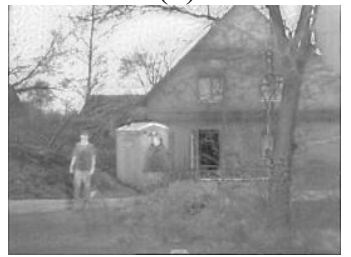

(h)

Fig. 2. Source image 'two men' and the corresponding fused images: (a) infrared source image; (b) visible source image; (c)-(h) fused images obtained with LPT-based, DWT-based, CT-based, NSCT-based, NSSTbased and our fusion algorithms, respectively.

As seen from Fig. 2, all of the fused images highlight the targets and preserve well the detailed structural information. However, the images depicted in Fig. 2c-g fail to describe correctly the background information on the sky, since the appropriate spectral information has been distorted. The results obtained with our algorithm (Fig. $2 \mathrm{~h}$ ) are successful as far as possible in preserving the original information. To compare the images in much detail, Fig. 3 depicts the detailed information about an enlarged local region of the fused images (see the location of the region shown in Fig. 2a). It is evident that the images displayed in Fig. 3c-g reveal very similar 
characteristics, whereas the structural information in panel (h) is smoother. Loosely speaking, the appearance of the right person in Fig. $3 \mathrm{~h}$ is roughly the same as that in the infrared source image, while the images in the other panels are 'contaminated' by a cabin seen in the visible source image. Therefore, one can conclude that the fusion algorithm proposed in this work tends to provide a better fusion performance.
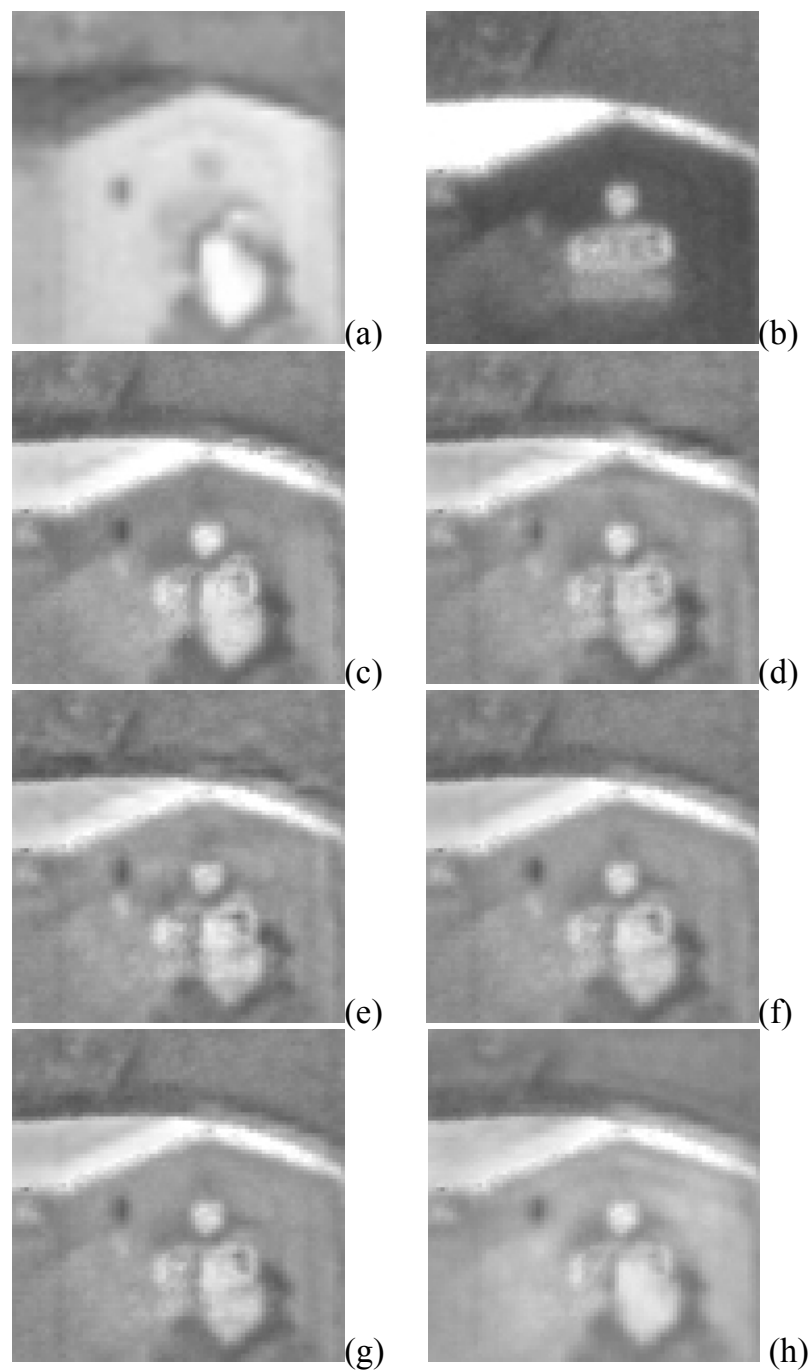

Fig. 3. Enlarged local regions of the fused images extracted from Fig. 2a-h, respectively.

The fusion results obtained for the other image sets with the six algorithms under our test can be subjectively compared using Fig. 4 . The results testify that our algorithm preserves both the thermal and texture information in the source image. The corresponding fused images look like sharpened infrared images, with clear and prominent targets. As a result, our approach can facilitate the automatic target detection.

We next provide the objective data of comparison of the six fusion algorithms, using the same five image sets. The results are listed in Table 1, where the best results are marked in bold. One can make sure that our algorithm provides the best performance, issuing from the majority of the fusion metrics. Fig. 5 visualizes the appropriate data of the comparison. Therefore we conclude that our fusion algorithm outperforms the other algorithms. 


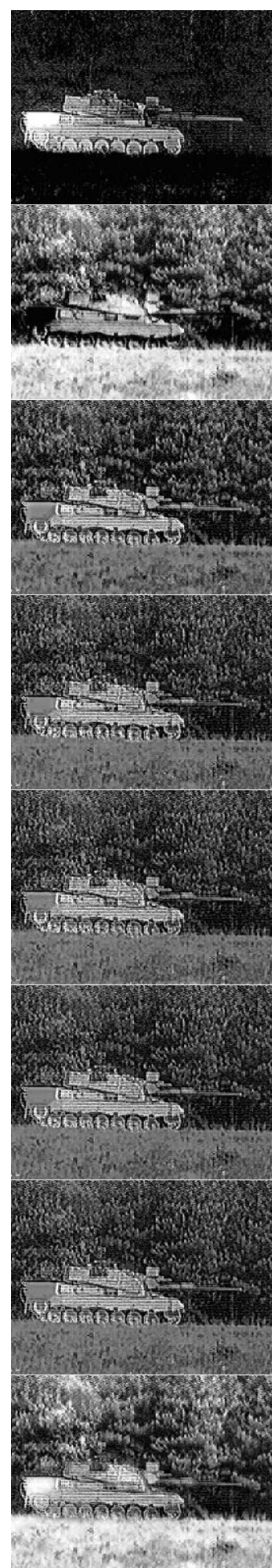

(a)

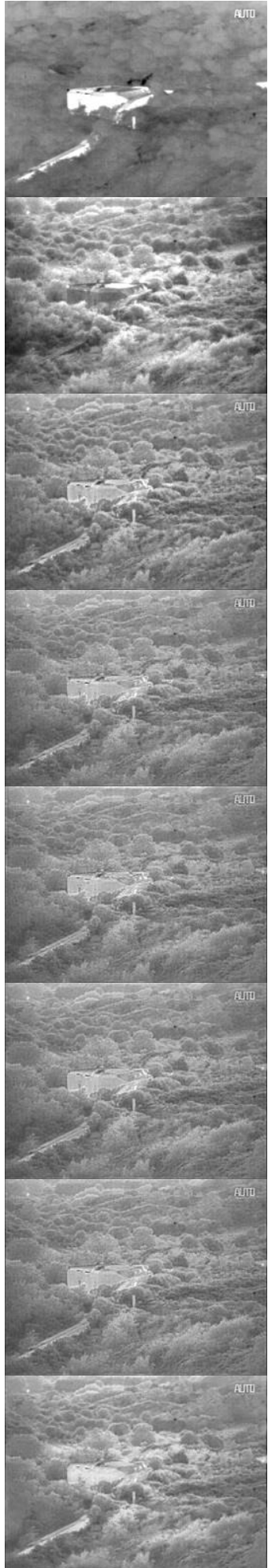

(b)

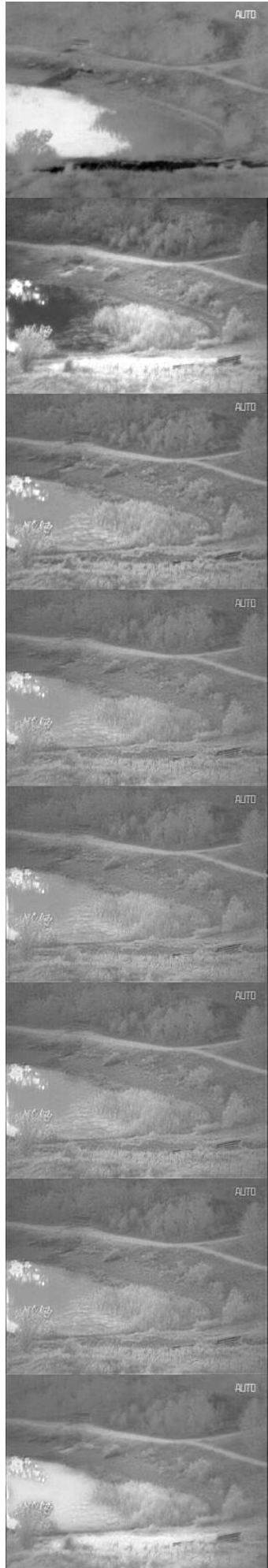

(c)

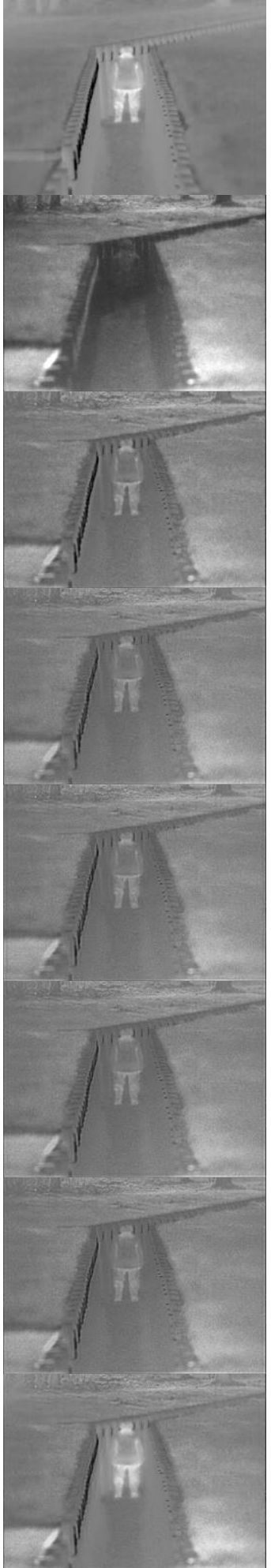

(d)

Fig. 4. Source images 'tank', 'banker', 'lake' and 'soldier', and the corresponding fused images: (a) 'tank', (b) 'bunker', (c) 'lake' and (d) 'soldier'. The first and second rows correspond to the infrared and visible source images, and the other rows correspond to the fused images obtained using LPT-based, DWT-based, CT-based, NSCT-based, NSST-based and our fusion algorithms. 
Table 1. Data of objective comparison of the six fusion algorithms, as obtained for the cases of five different testing image sets. The best results are marked in bold.

\begin{tabular}{|c|c|c|c|c|c|c|c|}
\hline $\begin{array}{c}\text { Image } \\
\text { set }\end{array}$ & Algorithm & $\begin{array}{l}\text { Average } \\
\text { gradient }\end{array}$ & $\begin{array}{l}\text { Informati } \\
\text { on } \\
\text { entropy }\end{array}$ & $Q_{0}$ & $\begin{array}{c}\text { Structural } \\
\text { similarity } \\
\text { index measure }\end{array}$ & $Q_{M I}$ & $Q_{T E}$ \\
\hline \multirow{6}{*}{$\begin{array}{l}\tilde{\Xi} \\
\Xi \\
0 \\
\vdots\end{array}$} & LPT & 0.0385 & 6.6877 & 0.3624 & 0.6264 & 0.2125 & 0.1916 \\
\hline & DWB & 0.0360 & 6.6041 & 0.3371 & 0.6223 & 0.2222 & 0.2097 \\
\hline & $\mathrm{CT}$ & 0.0361 & 6.5972 & 0.3211 & 0.6107 & 0.2144 & 0.2095 \\
\hline & NSCT & 0.0356 & 6.5796 & 0.3570 & 0.6376 & 0.2313 & 0.2178 \\
\hline & NSST & 0.0356 & 6.5791 & 0.3553 & 0.6371 & 0.2315 & 0.2177 \\
\hline & $\begin{array}{l}\text { our } \\
\text { algorithm }\end{array}$ & 0.0568 & 6.8867 & 0.4884 & 0.7011 & 0.3524 & 0.3045 \\
\hline \multirow{6}{*}{ 渮 } & LPT & 0.0830 & 7.4373 & 0.1466 & 0.1910 & 0.2565 & 0.3256 \\
\hline & DWB & 0.0777 & 7.3998 & 0.1640 & 0.2076 & 0.2430 & 0.3249 \\
\hline & $\mathrm{CT}$ & 0.0770 & 7.4086 & 0.1482 & 0.1893 & 0.2333 & 0.3241 \\
\hline & NSCT & 0.0752 & 7.4116 & 0.1641 & 0.2125 & 0.2601 & 0.3326 \\
\hline & NSST & 0.0750 & 7.4120 & 0.1629 & 0.2114 & 0.2596 & 0.3330 \\
\hline & $\begin{array}{l}\text { our } \\
\text { algorithm }\end{array}$ & 0.1067 & 7.9518 & 0.0878 & 0.1251 & 0.4003 & 0.3640 \\
\hline \multirow{6}{*}{ 㐫 } & LPT & 0.0470 & 6.9858 & 0.0344 & 0.3799 & 0.1985 & 0.1612 \\
\hline & DWB & 0.0412 & 6.8374 & 0.0553 & 0.4125 & 0.1876 & 0.1678 \\
\hline & $\mathrm{CT}$ & 0.0410 & 6.8392 & 0.0553 & 0.4112 & 0.1841 & 0.1633 \\
\hline & NSCT & 0.0406 & 6.8238 & 0.0562 & 0.4222 & 0.1932 & 0.1724 \\
\hline & NSST & 0.0407 & 6.8242 & 0.0548 & 0.4216 & 0.1930 & 0.1721 \\
\hline & $\begin{array}{l}\text { our } \\
\text { algorithm }\end{array}$ & 0.0464 & 6.9550 & 0.0689 & 0.4697 & 0.2667 & 0.2297 \\
\hline \multirow{6}{*}{ 造 } & LPT & 0.0386 & 6.6811 & 0.1729 & 0.6028 & 0.2313 & 0.2408 \\
\hline & DWB & 0.0365 & 6.6292 & 0.1729 & 0.6153 & 0.2423 & 0.2527 \\
\hline & $\mathrm{CT}$ & 0.0363 & 6.6334 & 0.1737 & 0.6129 & 0.2342 & 0.2481 \\
\hline & NSCT & 0.0362 & 6.6282 & 0.1849 & 0.6254 & 0.2469 & 0.2540 \\
\hline & NSST & 0.0362 & 6.6284 & 0.1850 & 0.6259 & 0.2467 & 0.2538 \\
\hline & $\begin{array}{l}\text { our } \\
\text { algorithm }\end{array}$ & 0.0529 & 6.9440 & 0.4039 & 0.7440 & 0.3563 & 0.2995 \\
\hline \multirow{6}{*}{$\frac{\bar{\theta}}{\bar{c}}$} & LPT & 0.0331 & 6.4895 & 0.1096 & 0.5296 & 0.2508 & 0.2341 \\
\hline & DWB & 0.0317 & 6.4417 & 0.0973 & 0.5284 & 0.2523 & 0.2339 \\
\hline & $\mathrm{CT}$ & 0.0314 & 6.4421 & 0.1005 & 0.5287 & 0.2440 & 0.2298 \\
\hline & NSCT & 0.0314 & 6.4372 & 0.1037 & 0.5335 & 0.2530 & 0.2333 \\
\hline & NSST & 0.0314 & 6.4367 & 0.1041 & 0.5338 & 0.2531 & 0.2335 \\
\hline & $\begin{array}{l}\text { our } \\
\text { algorithm }\end{array}$ & 0.0351 & 6.4689 & 0.2072 & 0.6762 & 0.3088 & 0.2573 \\
\hline
\end{tabular}



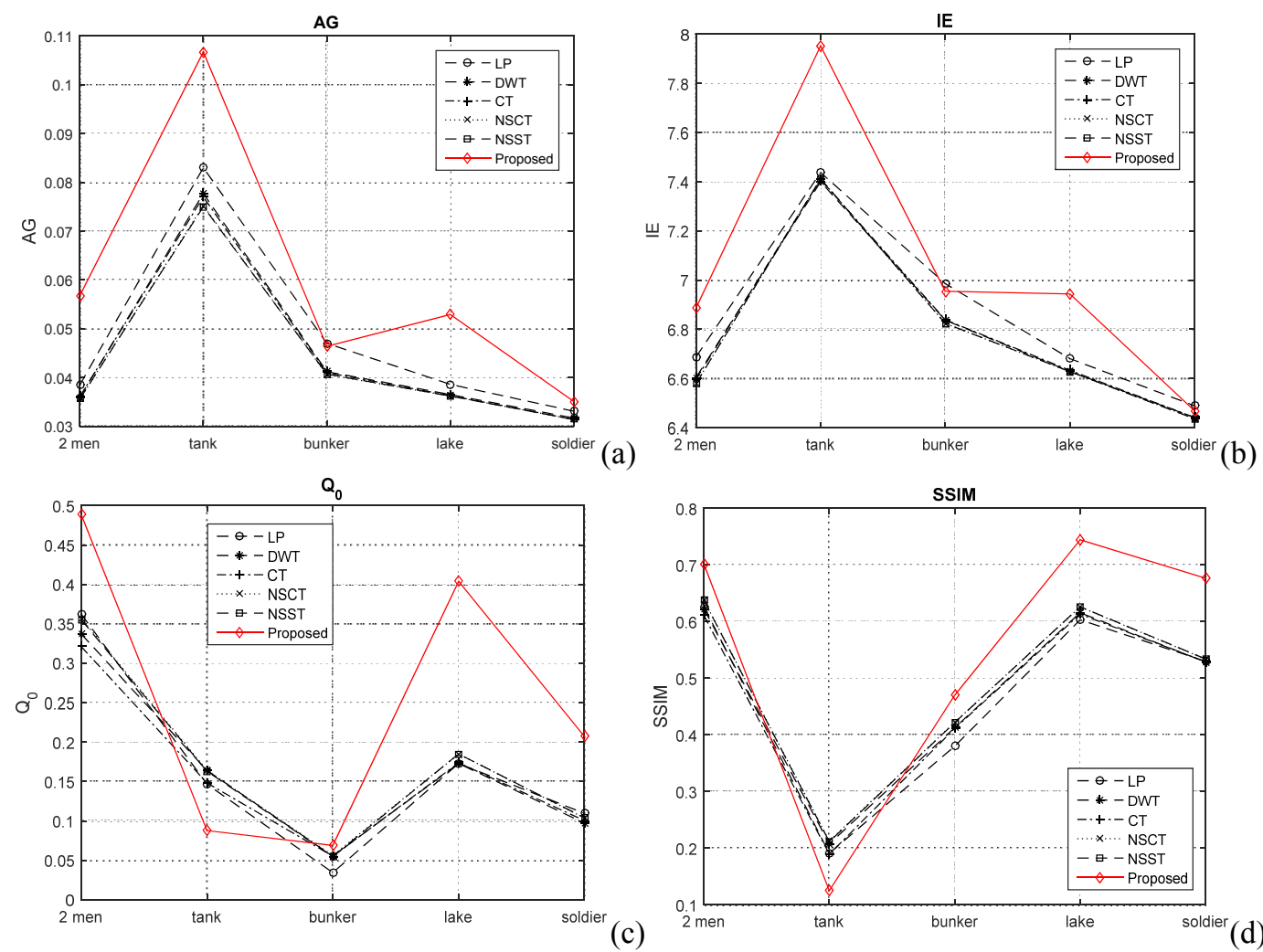

(b)
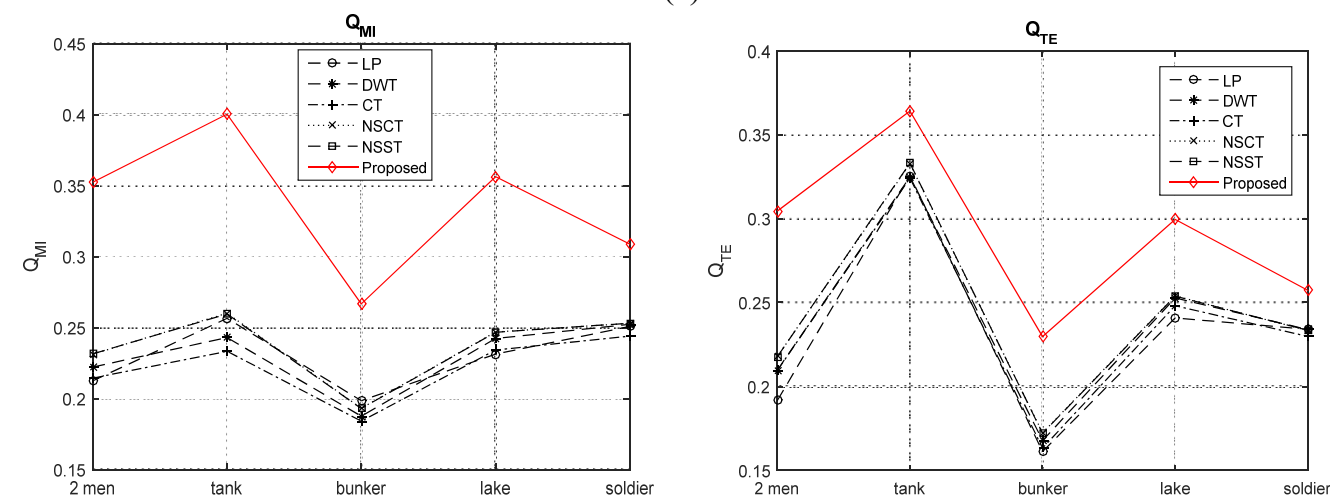

(d)

(e)

Fig. 5. Values of different fusion metrics: panels (a)-(f) correspond respectively to average gradient, information entropy, $Q_{0}$, structural similarity index measure, $Q_{M I}$ and $Q_{T E}$.

\section{Conclusion}

In this work, we suggest a new fusion algorithm based simultaneously on the SCDPT approach and the SUSAN edge detector. The fundamental reasons behind our choice are as follows. On the one hand, the SCDPT can describe more structural information and reduce the impact of misregistration because of its shift-invariance property. On the other hand, the SUSAN edge detector represents an approach of low-level image processing which is used for extracting the edge features. Then the local area of each pixel contains much structural information. By combining the above two approaches, we work out the combined algorithm for fusing the infrared and visible images. To implement the fusion process, we adopt the weighted-averaging fusion rule based on 
the local normalized energy for the low-pass sub-bands and the maximum-choosing fusion rule based on the SUSAN edge detector for the high-pass sub-bands.

The five sets of images have been used to check the performance of the algorithm proposed in the present work. The comparison with the experimental results obtained using five traditional algorithms known from the literature has been performed with both the subjective and objective standpoints. The data of the objective comparisons is based upon the five standard image quality metrics. Following from both the subjective evaluation data and the objective fusion metrics, we conclude that our algorithm is superior to the traditional algorithms.

\section{Acknowledgement}

This work has been supported by the National Natural Science Foundation of China (No. 61202464, No. 61472136 and No. 61772196), the Natural Science Research Program of Henan Educational Committee (No. 14A120012), and the Key Project of Hunan Province of China Social Science Fund (Program No. 2016ZBB006). Hunan Province of the China Social Science Achievement Evaluation Committee has also appraised and evaluated the research subject under the Program No. 2016JD05.

\section{References}

1. Ma Y, Chen J, Chen C, Fan F and Ma J, 2016. Infrared and visible image fusion using total variation model. Neurocomputing. 202: 12-19.

2. Bhatnagar G, Wu Q J and Liu Z, 2015. A new contrast based multimodal medical image fusion framework. Neurocomputing. 157: 143-152.

3. Zhang Q and Guo B, 2009. Multifocus image fusion using the nonsubsampled contourlet transform. Signal Processing. 89: 1334-1346.

4. Li S, Kang X, Fang L, Hu J and Yin H, 2017. Pixel-level image fusion: A survey of the state of the art. Information Fusion. 33: 100-112.

5. Nguyen T T and Oraintara S, 2008. The shiftable complex directional pyramid - Part I: Theoretical aspects. IEEE Trans. Signal Processing. 56: 4651-4660.

6. Zhang Q, Wang L, Li H and Ma Z, 2011. Similarity-based multimodality image fusion with shiftable complex directional pyramid. Pattern Recognition Lett. 32: 1544-1553.

7. Smith S M and Brady J M, 1997. SUSAN - a new approach to low level image processing. Int. J. Computer Vision. 23: 45-78.

8. TNO Image Fusion Dataset, https://figshare.com/articles/TNO_Image_Fusion_Dataset/1008029.

9. Gonzalez R C, Woods R E and Eddins S L, Digital image processing using MATLAB. Upper Saddle River, New Jersey: Pearson-Prentice-Hall (2004)..

10. Wang $Z$ and Bovik A C, 2002. A universal image quality index. IEEE Signal Processing Lett. 9: 81-84.

11. Wang Z, Bovik A C, Sheikh H R and Simoncelli E P, 2004. Image quality assessment: From error visibility to structural similarity. IEEE Trans. Image Processing. 13: 600-612.

12. Liu Z, Blasch E, Xue Z, Zhao J, Laganiere R and Wu W, 2012. Objective assessment of multiresolution image fusion algorithms for context enhancement in night vision: a comparative study. IEEE Trans. Pattern Analysis and Machine Intelligence. 34: 94-109.

Defa Hu, Hailiang Shi and Weijin Jiang. 2018. Infrared and visible image fusion based on shiftable complex directional pyramid transform and SUSAN edge detector. Ukr.J.Phys.Opt. 19: 199 - 210. doi: 10.3116/16091833/19/4/199/2018

Ukr. J. Phys. Opt. 2018, Volume 19, Issue 4 
Анотація. Ми пропонуємо алгоритм злиття інфрачервоних і видимих зображень, який базується на змішеному комплексному спрямованому пірамідному перетворенні та детекторі країв SUSAN. У зливанні коефіиієнтів піддіапазонів пропускання низьких частот використано правило зваженого усереднення, пов'язане з локальною нормованою енергією. У зливанні напрямних коефіцієнтів піддіапазонів пропускання високих частот використано детектор країв SUSAN для створення карти прийняття рішень $і$ керування процесом злиття зображень. Щоб зменшити складність обчислень, ми розраховуємо крайовий відгук SUSAN лише один раз безпосередньо для кожного вхідного зображення. Ми оцінювали запропонований алгоритм злиття на стандартному наборі зображень TNO Image Fusion Dataset $i$ порівнювали його з низкою традиційних алгоритмів злиття. Експериментальні результати засвідчили, щзо наш алгоритм є здійсненним і ефективним. Більше того, він перевершує традиџійні алгоритми з огляду і на суб 'єктивні очінки, і на об'єктивні метрики злиття зображень. 